\title{
Value-based focused global population health management
}

\author{
John F. Gibbs ${ }^{1}$, Ashley Newman ${ }^{2}$, Richard G. Stefanacci ${ }^{3}$ \\ ${ }^{1}$ Hackensack Meridian School of Medicine at Seton Hall University, Nutley, NJ, USA; ${ }^{2}$ Rutgers-Robert Woods Johnson, New Brunswick, NJ, USA; \\ ${ }^{3}$ Thomas Jefferson College of Population Health, Philadelphia, PA, USA \\ Contributions: (I) Conception and design: All authors; (II) Administrative support: None; (III) Provision of study materials or patients: None; (IV) \\ Collection and assembly of data: JF Gibbs, A Newman; (V) Data analysis and interpretation: All authors; (VI) Manuscript writing: All authors; (VII) \\ Final approval of manuscript: All authors. \\ Correspondence to: John F. Gibbs, MD, MHCM, FACS. Chair of Surgery, 1945 State Hwy 33, 4-Ackerman, Neptune, NJ 07753, USA. \\ Email: John.gibbs@hackensackmeridian.org.
}

\begin{abstract}
In 2018, approximately 18 million people worldwide were diagnosed with cancer and are predicted to double by 2040. The global quality chasm in improving health care worldwide requires "systems thinking" as the key to success. Aligning the goal around person-centered care captures the total needs of care of a population and not just disease categories. The integration of the Institute of Medicine's (IOM) six aims of quality termed "value-based focused" and population health management (PHM) provides all health care leaders grappling with improving the health care of the populations a framework for the communities they serve. In this context, the question becomes finding solutions to providing high quality, compassionate and patient-centered health care delivery. Over the last two decades, three paradigms have emerged; the six aims of quality, outcome-focused population health, and the "Quadruple Aim". We have termed the intersection of these concepts as Value-based focused Population Health Management (VBPHM). This review applies VBPHM across the geographic county and community levels in the United States. Specifically, we examine VBPHM at the county or county-equivalents and community levels within the United States. Lastly, the potential role of Community-based Participatory Research and it is applicability to our framework is discussed. VBPHM can comparably be applied globally to improve population health, especially in preventing and treating cancer better.
\end{abstract}

Keywords: Population health management (PHM); gastrointestinal cancer (GI cancer); triple aim; institute of medicine (IOM)

Submitted May 24, 2020. Accepted for publication Jul 09, 2020.

doi: 10.21037/jgo-2019-gi-10

View this article at: http://dx.doi.org/10.21037/jgo-2019-gi-10

\section{Introduction}

The growing burden of cancer worldwide, directly and indirectly, impact a population's health. Indirectly, cancer taxes many country's abilities to provide adequate care for their populations. The World Health Organization $(\mathrm{WHO})$ predicts that 1 in 5 people will have cancer in their lifetime (1). An estimated 18 million people worldwide were diagnosed with this disease, and the rate is predicted to double by 2040 (2). The incidence of gastrointestinal (GI) comprises approximately one-third of all cancers worldwide
$[5,287,868]$ (Table 1). This rise in cancer rates is also occurring in low to middle-income countries.

Our impacted citizens are left struggling to maximize their contributions to society. Premature deaths resulting from advanced disease leads to the loss of "human capital" (3). The WHO identifies the "substantial global heterogeneity" of leading cancer types from differences in risk factors to life expectancy (4). With a globally connected population, we recognize that "diseases do not need passports to cross borders". The need for a unified approach to managing cancers globally is essential (5). In response 
to a mandate from the World Health Assembly on cancer prevention and control and prevention, the WHO's 2020 Report on Cancer provides recommendations on setting priorities, investing wisely, and giving care (Table 2). A global call to action is recommended to implement effective, feasible cancer management interventions, ensuring highquality value-based care (1).

The "quality chasm" initially reported in the United States is, in fact, a global "tipping point" (6). As we are still facing severe health care delivery gaps worldwide, the question we face is "bow to maximize health care benefits while minimizing cost?" In "Crossing the Global Quality Chasm: Improving Health Care Worldwide", Berwick et al. [2018] assert that "systems thinking" is the key to success $(7,8)$. Aligning the goal around person-centered care captures

Table 12018 Global Gastrointestinal Cancer Incidence (2)

\begin{tabular}{lccc}
\hline Rank & Cancer & New cases [2018] & \% of all cancers \\
\hline 1 & Colorectal & $1,800,977$ & 10.6 \\
2 & Stomach & $1,033,701$ & 6.1 \\
3 & Liver & 841,080 & 5.0 \\
4 & Esophagus & 572,034 & 3.4 \\
5 & Pancreas & 458.918 & 2.7 \\
6 & Oral cavity* & 354,864 & 2.1 \\
7 & Oropharynx & 92,887 & 0.5 \\
8 & Hypopharynx & 80,608 & 0.5 \\
9 & Salivary Glands & 52,799 & 0.3 \\
\hline
\end{tabular}

*, the oral cavity is the first part of the digestive system. the total needs of care of a population and not just disease categories. The WHO identified several populationbased, highly cost-effect intervention options termed "Best Buy", recognizing the need to deliver quality care within budget constraints (9). Given resource constraints, Roberts and colleagues "Five control knobs" involving financing, payment, organization, regulation, and behavior is a useful model incorporating the necessary tradeoffs to achieve the optimal performance goals of a target population (10). A valued-based approach provides the necessary skill set to coordinate global cancer care delivery.

The institute of medicine (IOM) committee's report "Crossing the Quality Chasm: a New Health System for the 21 st Century" emphasized transparent, evidence-based, and patient-centered care (6). This document recommended six aims of quality (safe, timely, effective, efficient, equitable, and patient-centered) for improving health care, referred to by the acronym "STEEP". Shortly after that, populationbased health emerged as unifying the movement. Morris' [1957] classic work, Uses of Epidemiology, provides an elegant definition of a population as "the study of health and disease of populations and groups concerning their environment and ways of living... The population may be of a whole country on any particular sector of it [as delimited by people] environment, their living conditions, and special ways of life." (11). Thus, there are two conditions to be a population: (I) there is more than one individual, and (II) individuals share at least one common characteristic. Additionally, a population shares common characteristics, most often a specific location they inhabit. A particular town, village, city, state, or country and all those who live within that geographically defines the boundary of the population.

Table 2 World Health Organization 2020 cancer recommendations (1)
(I) Activate political will, strengthen governance, and make a cancer control plan founded
(II) Identify priorities that are feasible, evidence-based, and financeable
(III) Focus on WHO "Best Buys" for Noncommunicable Disease (NCD) primary preventions
(IV) Prioritize and invest in early diagnosis
(V) Implement effective, feasible cancer management interventions, ensuring high-quality value-base care
(VI) Strengthen information systems to improve planning and accountability
(VII) Fund priorities in cancer interventions and ensure protection
(VIII) Build capacity through cancer centers and networks linked to strong primary care
(IX) Optimize the workforce and access to reliable sustainable medicines and other products
(IX) Engage communities and civil society to achieve cancer control together 
The Patient Protection \& Affordable Care Act (PPACA) health care reform law enacted in March 2010 has accelerated the shifts from volume to value-based care delivery (12). Two essential areas emanating from the PPACA are the focus on community-based initiatives and population-based payment models. The WHO defines "communities as groups of people that may [or may not] be spatially connected, share common interests, concerns, or identities." The PPACA also resulted in the rise of population risk-based payment, including global capitation. Understandably, there is considerable concern among stakeholders. However, improved care delivery models are emerging for managing populations.

Over the last two decades, three transforming reports have influenced health care reform in the United States framing this review. First, the definition of "Population Health" by Kindig \& Stoddart, as "the health outcomes of a group of individuals [population] including the distribution of such outcomes within the group [population]" $(13,14)$. Second, "The Quadruple Aim" focus on delivering health care by improving the experience of care and outcomes while reducing per capita costs (15-17). Lastly, STEEP emphasis on delivering patient-centered valued care. We interpret the intersection of these three paradigms as valuebased care delivery, improving population-level outcomes. This review introduces the integration of the IOM's six aims of quality (STEEEP) termed "value-based focused" and population health management (PHM) aimed at delivering patient-centered value-based care. Nash [2012] provides a viewpoint of population health as the roof of a house supported by "Four Pillars": chronic care management, quality and safety, public health, and health policy (Figure 1) (18). He further posits that the incorporation of these concepts sets the foundation for achieving population health and strategies. Many questions remain during this "new reality", however, we contend that this model best positions providers and health systems to meet populationlevel outcomes.

For this review, we provide an operational model of value-based population health management (VBPHM) for all health care leaders grappling with improving the health care of the populations they serve. While addressing the health of an entire community is essential, VB-PHM requires focused efforts to remain cost-effective while producing real improvement in health care delivery. Taking an oncologist management approach stage within the continuum of care, we further subdivide management into episodes of care (Figure 2) (1).
Segmentation allows an organization to identify cohorts of patients that would benefit most. The concepts of VB-PHM can improve the health and wellness of our patients and communities, especially under the condition of limited resources. The application of geographic divisions at county and community levels within the United States illustrates VB-PHM, similar to other geopolitical divisions worldwide.

\section{Health factors/determinants of health}

Population health refers broadly to the distribution of health outcomes within a population, the health determinants that influence the distribution, and the policies and interventions that affect those determinants (13). Kindig and Stoddard [2003] articulated population-level outcome measures as how long (years of potential life lost) and well (quality of life) individuals among a population live (13). Thus, why does a population have the existing distribution of a particular risk? $(19,20)$. Addressing health factors as the independent variables influencing the health outcomes (dependent variable) is a framework to evaluate it.

The Dahlgren-Whitehead [1991] "Rainbow Model" separates the population health determinants into layers of general socio-economic cultural and environmental conditions; living and working conditions; social and community networks; individual lifestyle facts; and age, sex, and heredity factors (21). Researchers from the University of Wisconsin Population Health Institute collated disaggregated population health data for analyzing health outcomes among county geographical levels within their state associated with health outcomes (22).

Along with the growing awareness of Social Determinants of Health (SDoH), there is some confusion with its relationship to social risk factors and social needs. The WHO definition of SDoH has "the conditions which people are born, grow, live, work, and age" illustrates the multiple levels it impacts (23). SDoH, thus, has both a positive connotation rather than, merely, a negative one. Additionally, a country's structural factors influence the distribution of resources and the daily lives of a population. Individual-level adverse SDoH referred to as social risk factors, are specific adverse social conditions associated with poor health. Social risk factors are not synonymous with behavioral risk factors nor social needs $(24,25)$. However, social risk factors do influence individual behavior. Social needs involve the individual's role in identifying and prioritizing a social intervention. An individual may be 


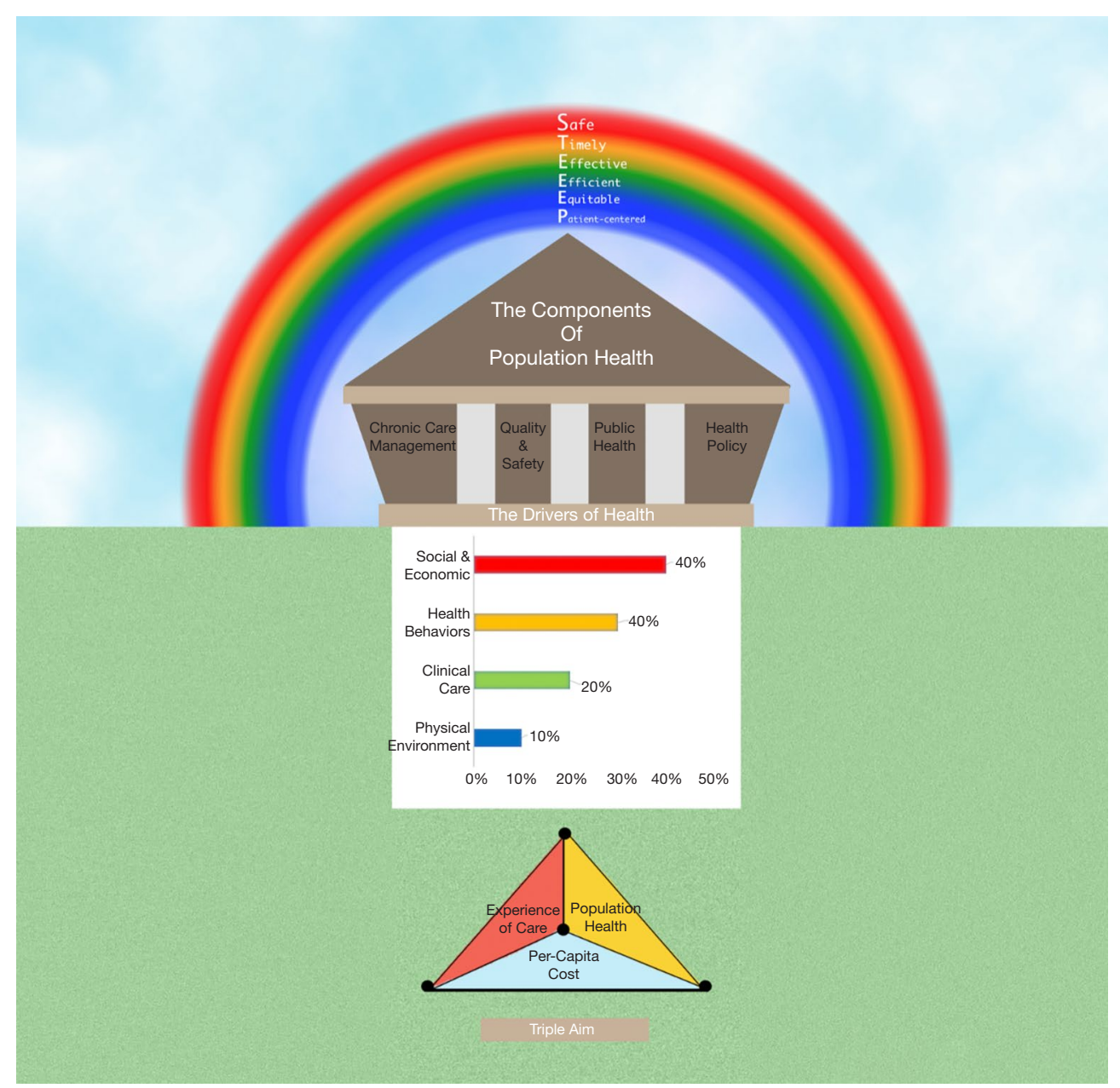

Figure 1 Value-based Focused Population Health Management is the intersection with the IOM's Six Aims of Quality (The "STEEEP" Rainbow), the Four Pillar's supporting Population-level health outcomes, and the "Triple Aim" care delivery principles (Improving the experience of care, improving population health, and reducing per capita cost).

assessed to have several social risk factors but may only be requesting assistance with one need. What matters is the context of a patient's life rather than presumed clinical need?

\section{VB-PHM and the continuum of care}

Hodach [2016] emphasizes that effective PHM is dependent on engaging patients and coordinating care across the settings and over time. The concept of caring for entire populations continuously has become a focus of all health care stakeholders (26). VBPHM focus on a populationcentric continuum of care includes increasing coordination of care, improved access to care, specialized care for patients with severe or complex conditions, or support for patients with nonmedical barriers to good health care.

Oncologists are accustomed to managing patients across the phases of cancer care from prevention to the end of life (Figure 3). Guidelines and staging processes are reflective of oncologic care through various episodes of care. Allen advocates the grouping of populations according to their conditions, severity of illness, demographic qualities, location in identifying risk levels aids in leveraging resources to improve care and outcomes (27). Hordach presents a three-step roadmap for PHM (26). The first step is beginning with the risk stratification of a population to identify which patients have the most significant health risks. Second, health care systems must re-engineer their processes to be safer and more efficient. Thirdly, the integration of health information technology is essential to 
the management process.

Appropriate VB-PHM based care should include transparency and accountability with a focus on populations and subgroups and the episodes or continuum of care (EOC). Ten percent of Americans account for $65 \%$ of total

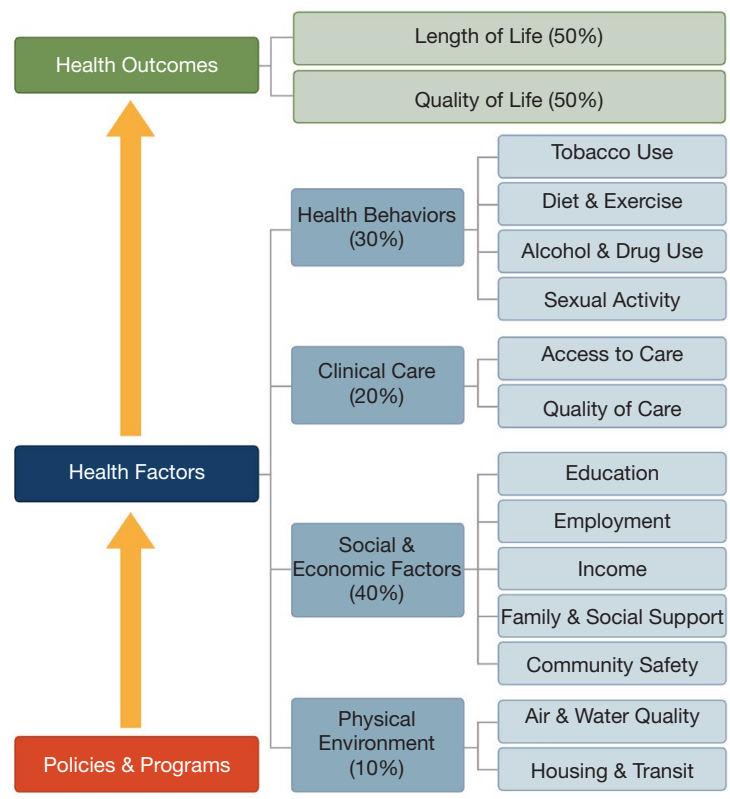

Figure 2 The RWJF-UW County Health Rankings \& Roadmaps Program aims to improve health outcomes and close health gaps. It provides an interactive model for health and community leader to evaluate county-level rankings within the United States and to identify areas for community improvement. (Reproduced with permission) (https://www.countyhealthrankings.org/explorehealth-rankings/measures-data-sources/county-health-rankingsmodel). health care spending (28). Although some would argue for directing resources for most patients along the spectrum of care rather than the extremes, this review focuses on the position of biologic rather than economic debates. Four biologic cohorts consisting of single acute illness, earlyonset chronic condition, full onset chronic disease, and complex (including catastrophic) EOC compartmentalizes care management approaches. The staggering cost involved in caring for the subgroup of our population with full onset chronic conditions (e.g., cancer, stroke) or complex (aka catastrophic) EOC is well documented (28). When evaluating any venture that has the best potential for success and sustainability, we propose evaluating its reasonability, feasibility, and practicability. Patient-centered specialty practice (PCSP) focusing on cancers has collaborated with patient-centered medical homes $(\mathrm{PCMH})$ to provide better patient care (28-30). This partnership offers an opportunity for the development of clinical protocols to standardize the care of patients. Multidisciplinary care (MDC) of cancer patients through tumor boards and the National Comprehensive Cancer Network (NCCN) guidelines are a critical component of delivering effective cancer care. A recent study evaluated fourteen cancer center's MDC implementation level's impact (low to high) across seven assessment areas (care planning, physician engagement, coordination of care, infrastructure, financial, clinical trials, and medical records) (29). The investigators demonstrated that "a high level of MDC cancer care coordination was associated with an increased likelihood of multimodality therapy and guideline-adherent care." Advances in the interoperability of EHR platforms, among multiple software applications, will accelerate the transition from silo care to coordinate population-based care (27).

\section{Value Based Focused Care}

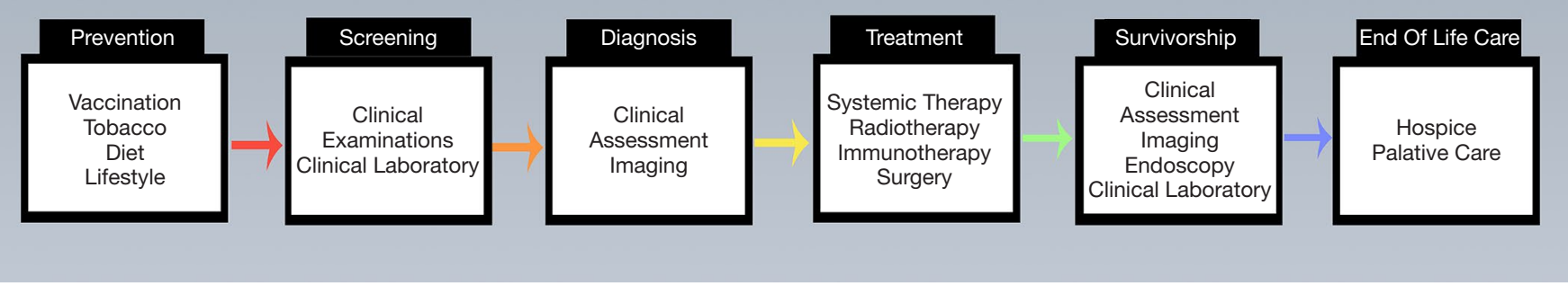

Figure 3 Interventions across along the "cancer continuum" from prevention to survivorship or end-of-life care (WHO 2020). 


\section{Identifying health gaps with counties and communities}

Addressing health behaviors and social/economic are considered the top priorities in PHM. In the United States, geographic grouping by county or countyequivalents is regarded a stable framework. Improving the health outcomes of a group of individuals, including the distribution of such results within the group, requires an evaluable data registry. Worldwide, a country is considered a standard unit of geographic analysis. Unfortunately, the creation of geographic divisions in other countries is complex, limiting consistent the collection of accurate registry data (2). In the United States, the county or countyequivalent, the geopolitical unit, will be utilized as a model for identifying gaps in health care. Currently, there are approximately 3,250 counties or county-equivalents in the U.S. that are subdivided into cities and communities (31). The County Health Ranking \& Roadmap (CHRR) developed in partnership between the University of Wisconsin Population Health Institute and the Robert Woods Johnson Foundation (UW-RWJF) collect countylevel health status data from across the United States. CHRR model depicts health outcomes resulting from multiple health factors or determinants. Geographic variations exist across the United States, including unacceptable disparities in morbidity, mortality, and risk factors $(32,33)$. The model provided by the UW-RWJF County Health Ranking and Roadmaps (CHRR) illustrates the division of health factors into four categories: Health behaviors (30\%), Clinical care (20\%), Social \& economic factors (40\%), Physical environment (10\%) (Figure 2). Although there is a debate with summating these factors to $100 \%$, the model is useful for motivating all stakeholders to address gaps of care in their communities $(32,34)$. The increased attention to improving health in all communities across the United States utilizing the CHRR database is limited.

Community Health Needs Assessment (CHNA) with an associated implementation strategy following CHRR allows the development of policies at the closest point of impact $(12,35-38)$. The combinations of the county or countyequivalents with community-level reflect the healthcare boundaries of the decision-making approach in the United States. The global community that has similar boundary distinctions can apply this model within its country. A CHNA is developed every three years as mandated by the PPACA. For example, the State of New Jersey is composed of 21 counties. Most counties have subdivisions, which may include municipalities and unincorporated areas. Nineteen cities comprise Cumberland County (CU) (39). The poverty rate is $18.8 \%$ of its 154,952 population [2012]. Household size is 2.8:1 with a median household income of $\$ 50,000$. CHRR ranks CU 21 of 21 counties in New Jersey (County Health Rankings 2019). CU's four affluent cities provide a striking variation in the percentage of the population below the poverty line. Selecting the priorities of needs for the more impoverished areas within $\mathrm{CU}$ requires the incorporation of critical stakeholders to pick and rate the top issues identified.

Once community health needs are rated, a prioritization process is undertaken. The modified Hanlon prioritization rating method, the "PEARL" Test, is popular among several other models (40). PEARL denotes the following feasibility factors: propriety, economics, acceptability, resources, and legality. Propriety addresses whether a program for the health problem is suitable. Does it make economic sense to solve the problem and uncover potential deleterious consequences? Will the program be acceptable to the community? Are resources and funding available or potentially available for a program? Finally, do current laws support program activities? In the case of C.U., the areas of needs in descending order were adult and childhood obesity, substance abuse (drug, alcohol, and tobacco), mental and behavioral health, and access to care. A strategy at improving healthy food choices was selected.

\section{Upstream versus downstream policies and interventions}

A common metaphor, in population and public health, describes a contaminated water source influencing inhabitants' downstream in planning policies and interventions (41). The inhabitants of an affected village ("downstream") devise methods to treat water by boiling or filtering when the issue is the primary source of contamination "upstream". National policies, for example, tobacco taxes, are aimed at addressing upstream causes. Downstream interventions, as depicted by the illustration, tend to concentrate at the individual level. Social determinants of health (e.g., unhealthy lifestyle behaviors) are associated with obesity, cardiovascular disease, type 2 diabetes, and cancer. These behaviors can be improved by eliminating tobacco products, eating healthy, increasing 
Table 3 Social determinant of health assessment tools by IOM domains

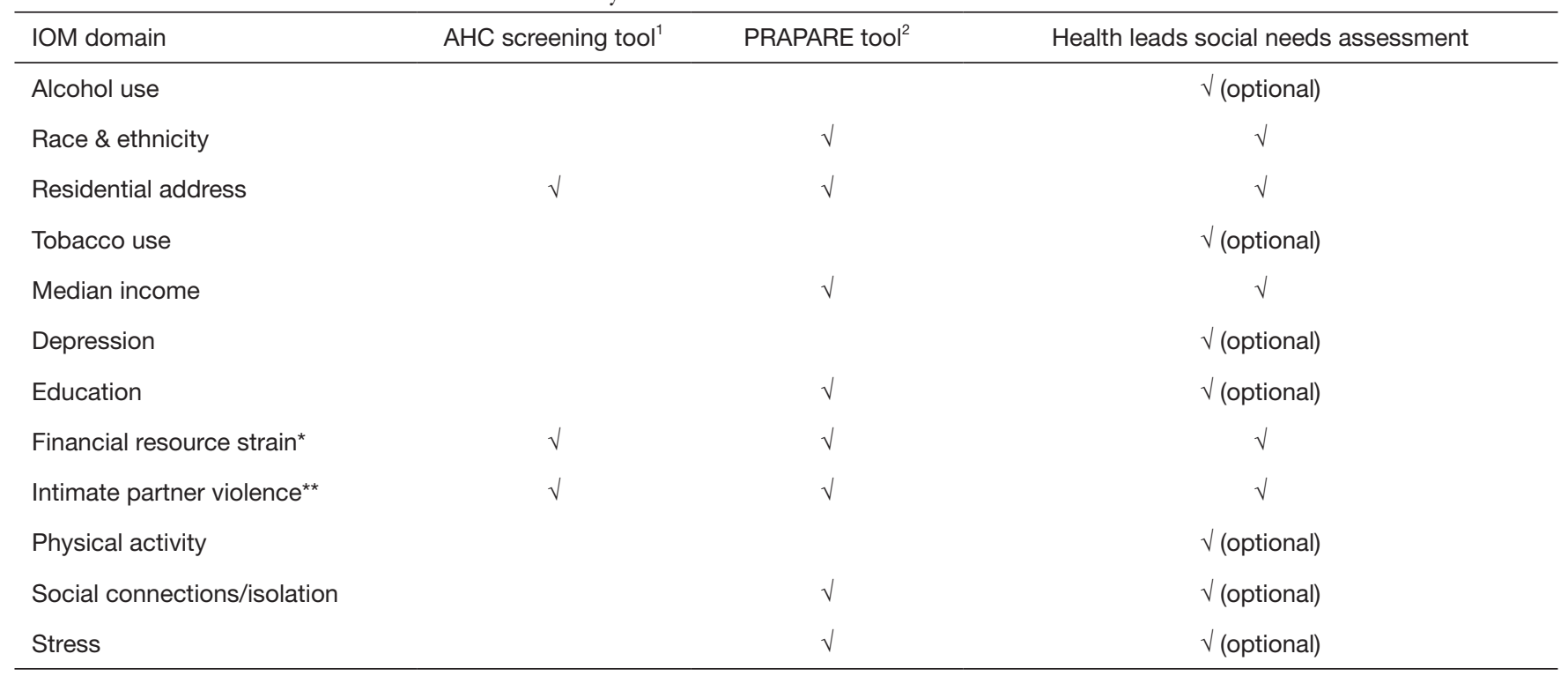

${ }^{*}$, includes food and housing scarcity; ${ }^{* *}$, may include interpersonal safety, safe environment, and/or exposure to violence. ${ }^{,}$, Accountable Health Communities; ${ }^{2}$, Protocol for Responding to and Assessing Patients' Assets, Risks, and Experiences. [Modified, reproduced with permission AccessHealth Spartanburg; (42)].

physical exercise, and drinking alcohol in moderation. A vital population health precept stresses focusing on the term "upstream medicine". Specifically, addressing the problem by turning it off at the source.

Assessment tools for screening $\mathrm{SDoH}$ are emerging (Table 3) (42). Protocol for Responding to and Assessing Patients' Assets, Risks, and Experiences (PRAPARE) screening tool for Social Determinants of Health in Populations is a standardized assessment. The IOM Committee on the Recommended Social and Behavioral Domains and Measures of Electronic Health Records (EHR) recommended that at minimum, ten patients-reported social and behavioral domains and one neighborhood/ community-level domain be documented in EHRs (43). The recommendations established a much-needed benchmark for prioritizing SDOH categories for patient assessments.

Integrating an $\mathrm{SDOH}$ tool with patient health records can be costly and time-consuming, and may require a significant investment in data system upgrades. As a result, some providers may opt for more "low-tech" methods, such as paper and pencil surveys. They have the resources to embed the tool electronically. For example, before adding its $\mathrm{SDOH}$ assessment into its care management software, AccessHealth Spartanburg collected information and tracked referrals using paper records (41).
Organizations administering SDOH assessments often establish workflows to track patient needs and referrals. Standardizing the process of screening patients and referring them to services, allow the care team to better understand their roles and responsibilities. Provider workflows typically include: time frame for administering an assessment (e.g., during intake following the first appointment), care team member(s) responsible for conducting evaluations and subsequently making referrals, and tracking of necessary referrals and follow-up.

Communicating appropriately with patients about SDOH. Building an adequate referral network. Integrating electronic assessment tools and resource inventories. Breaking down silos between health and social service organizations

VBPHM interventions, including policies, target both upstream and downstream levels (44). Investigators from the Institute for Healthcare Improvement provide a framework for measuring upstream and individual-level health determinants with health outcomes (Figure 4) (45). They demonstrate the role of integrated health care with individuals to achieve health promotion and disease progression. An example of upstream and individual-level intervention is targeted for alcohol control in the states. Among the younger population, excessive alcohol intake leads to increased traffic accidents, violence, and weak social 


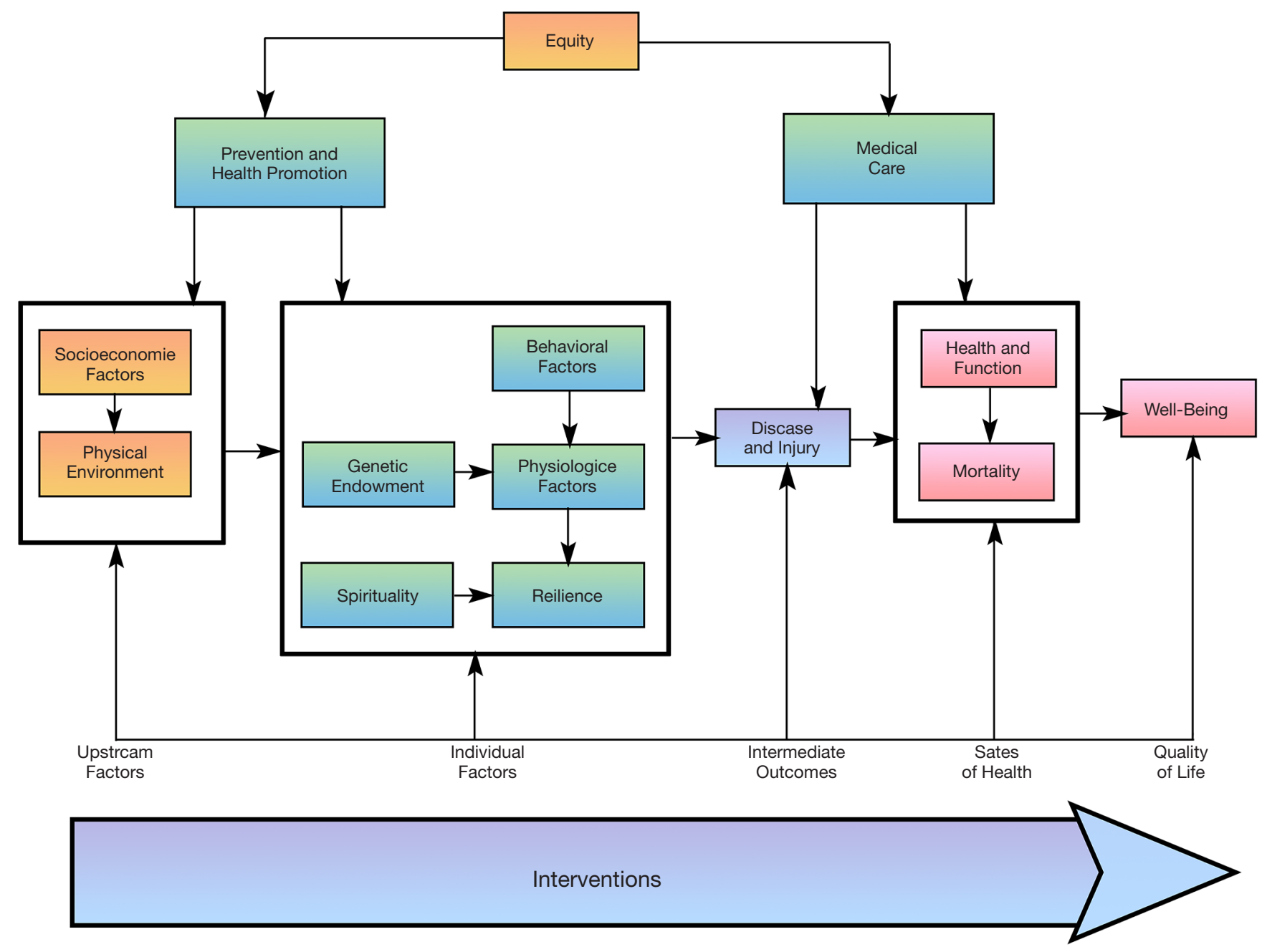

Figure 4 The IHI Population Health Composite Model provides a framework for measurement of health determinants (upstream and individual factors) and health outcomes. In this model, the IHI distinguishes between intermediate outcomes (disease burden and injury) and health outcomes (states of health). (“Adapted from www.IHI.org with permission of the Institute for Healthcare Improvement, @2020.”)

interactions.

Additionally, chronic excessive alcohol can lead to endstage liver disease and alcohol-associated hepatocellular carcinoma (46). A recent study has found that the number of alcohol-related deaths has increased by $50.9 \%$ from 1999-2017 (47). National policies, such as increased taxes and stiffer laws aimed at underage drinking, have reduced the risk of untoward alcohol-associated effects.

The IOM committee report found that the military's substance problems are a public crisis (48). In response, the military leadership identified harmful/hazardous alcohol use as an area for intervention. The Alcohol Use Disorders Identification Test (AUDIT) is a 10-question screening tool developed by the WHO to assess alcohol consumption, drinking behaviors, and alcohol-related problems (49). Although shortened versions of AUDIT are available, the central premise is to identify harmful alcohol consumption among high-risk populations. Beste et al. [2015] reported an increased rate of cirrhosis and hepatocellular carcinoma among U.S. veterans from 2001-2013 (50). The high price of chronic hepatitis C in this veteran population contributes significantly to the increased rate of HCC. However, these men were found to have both chronic hepatitis $\mathrm{C}$ and alcohol abuse making it challenging to distinguish attribution of ALD. In an autopsy study of male U.S. veterans, steatohepatitis, 
Table 4 Modified principles of community-based participatory research (56,57)

(I) Recognize the community as a unit of identity

(II) Build on the strength and resources within the community

(III) Facilitate a collaborative, equitable partnership in all phases of research, involving and empowering shared decision-making and governance

(IV) Foster co-learning and capacity building among all partners

(V) Integrate and achieve a balance between knowledge generation and intervention for the mutual benefit of all partners

(VI) Focus on the local relevance of health problems related to the multiple determinants of health

(VII) Utilize system thinking tools such as the IHI's Model for Improvement PDSA iterative process

(VIII) Disseminate all results for joint interpretation

(IX) Development long-term partnership and commitment to sustainability

(X) Address culturally sensitive conflicts promptly

(XI) Assure research rigor and validity and agreement of relevance to the community

chronic hepatitis $\mathrm{C}$, and cirrhosis were found to have an increased rate of primary liver cancer (51). They conclude that the increased rate of steatohepatitis and high levels of alcohol abuse in this cohort were likely secondary to alcoholic liver disease in most veterans. Larson et al. [2014] reported that 52,990 of 333,803 (15.9\%) post-deployment active duty army service members have behavioral/mental health or alcohol issues (52). Surprisingly, 29,711 service members were not referred ("missed opportunity") for intervention. There is a higher incidence of binge drinking among service personnel, potentially influencing their health and performance.

The overarching goal of VBPHM is to create "healthy people in health [global] communities" (18). The IOM describes a community as a group of people who share some or all of the following: geographic boundaries; a sense of membership; culture and language; conventional norms, interests, or values; and common health risks or conditions (20). Despite the awareness of the problem of health inequalities in the last Century, there remains a global issue. Health inequalities refer to the differences in health within a group or community. Conversely, health inequity or disparity has been a social injustice (53). The WHO Commission on Social Determinants of Health evaluated and provided recommendations on health inequities. Community or group-level differences determine social inequalities. However, past negative experiences and mistrust, such as the Tuskegee syphilis project, have led to distrust. As researchers have begun to engage communities with poorer health and living conditions, they have encountered an old century quote: "nothing about us without us." (54). Community-based Participatory Research (CBPR) is defined as "a systematic inquiry, with the collaboration of those affected by the issue, for purposes of education and taking action or effecting change" (55).

The success of CBPR is dependent on developing a shared partnership between academic researchers and community participants (55). A community must be considered as a unit of identity (56). In this Framework, it is the social interactions and community connectives that unify these populations. From a geographic perspective, the community may be a defined neighborhood or "dispersed ethnic group" with a shared sense of collective identity. CBPR attempts to engage and collaborate with these communities of identity (56). Academic researchers have learned that successful CBPR must view the communities as partners rather than subjects of research interventions. As in all partnerships, there are stages of engagement ranges from minimal through to complete. The foundation of trust is critical to developing a long-term relationship. During this transition, conflicts are inevitable.

Salsberg et al. [2014] describe approaching all members of a CBPR study as knowledge users from within a broader environment that may use, benefit, or be affected by the study (57). A successful CBPR study is dependent on the relationship of the study researchers and integrated knowledge users (IKU). The IKUs selected among the affected community, based on their best fit for the study. Thus, IKU are representative of the 
population who are partners in generating, presenting, and disseminating the results. IKU are those knowledge users who are actively involved in the knowledge production of the given study.

Ten fundamental principles to achieve a successful outcome in CBPR have been provided (Table 4) (56). Within partnerships, there is always the potential for conflict. A written partnership should be vetted and agree upon during the early phase of the project. Additionally, a supermajority ("70\% rule") can be embedded. The tenet is that everybody must agree that they can live with the decision, even if it is not their preferred choice (57). Several of these principals are illustrated in the following studies. One study aimed was to improve the low rate of colon screening in an American Indian community using a focus group intervention (58). Only 34.4\% of American Indians were up to date with colorectal screening guidelines, including fecal occult blood tests or endoscopy. The Focus Group, led by a community project moderator research representative, allowed for a safe environment for improved community participation. Researchers and community members collaborated to create a moderator guide for the community moderator led focus group sessions. The authors conclude that their methodology resulted in a successful process for conduction focus groups. Smith [2012] evaluated the use of community health workers in an African American (AA) community to improve colon cancer screening (59). AA's are nearly $50 \%$ more likely than whites to die from colorectal cancer. Community health workers are defined as individuals who should be members of and selected by the communities where they work. They should be answerable to the communities for their activities and supported by the health system but not necessarily a part of its organization (WHO). CHWs were involved during each phase of the 10-year Community Intervention Trial. The CHW were IKU and critical members of the intervention team, for example, selecting the group education model during the first phase of the study. CHW recommended the addition of brochures increasing the acceptability of the intervention and community engagement. Reflection led to the identification of maintaining core elements (fidelity) while being adaptable to noncore elements of the intervention.

Consolidated Framework for Implementation Science (CFIR) is a widely used structure for planning of evidencebased practices to implementation (60). Determinant frameworks are useful for understanding or explaining influences on the implementation impact of behavioral change or health outcomes at the individual or population level. This Framework includes several constructs in five domains (Figure 5). The first domain evaluates aspects of the intervention that may facilitate or impede the implementation process. The characteristics of the intervention may include complexity or costs. The second domain, outer setting, contains factors that can hinder the implementation process or outcomes. Characteristics of the outer setting include external policies, financial incentives, or aspects that may difficult to negotiate. The third domain, inner setting, conceptualizes factors that influence how processes or guidelines are implemented. Inner setting characteristics include the organization culture or climate, implementation climate, and organizational readiness for change. The fourth domain relates to the individuals or teams responsible for implementing the evidence-based practices, programs, or guidelines. The fifth area is the process of implementing the intervention. It includes a staged iterative process by which the implementation may occur.

Four stages are planning, engaging, executing, and reflecting/evaluating. The Consolidated Framework for Implementation Research (CFIR) and RE-AIM (Reach, Effectiveness, Adoption, Implementation, Maintenance) are two separate commonly employed planning and evaluation models planning translation of research into practice $(61,62)$. Both frameworks can be adapted to behavior, scientific, or pragmatic models. King et al. [2020] recent study demonstrate the benefit of CFIR in conjunction with RE-AIM's Adoption and Maintenance supporting the alignment of behavioral change. Both frameworks were adapted to a study examining the multilevel perspectives regarding outreach and engagement of three community stakeholders utilizing the Screening, Brief Intervention, and Referral to Treatment (SBIRT) tool for individuals at risk for substance abuse (63). Interestingly, the study investigator utilized CBPR and the CFIR domains and constructs to evaluate the implementation process. The modified CFIR and RE-AIM serves as an illustration of the benefit of incorporating both frameworks for planning future CBPR studies (Table 5).

\section{Futures perspectives}

With inequality in the spotlight these days, the causative upstream factors can have a significant ripple effect 


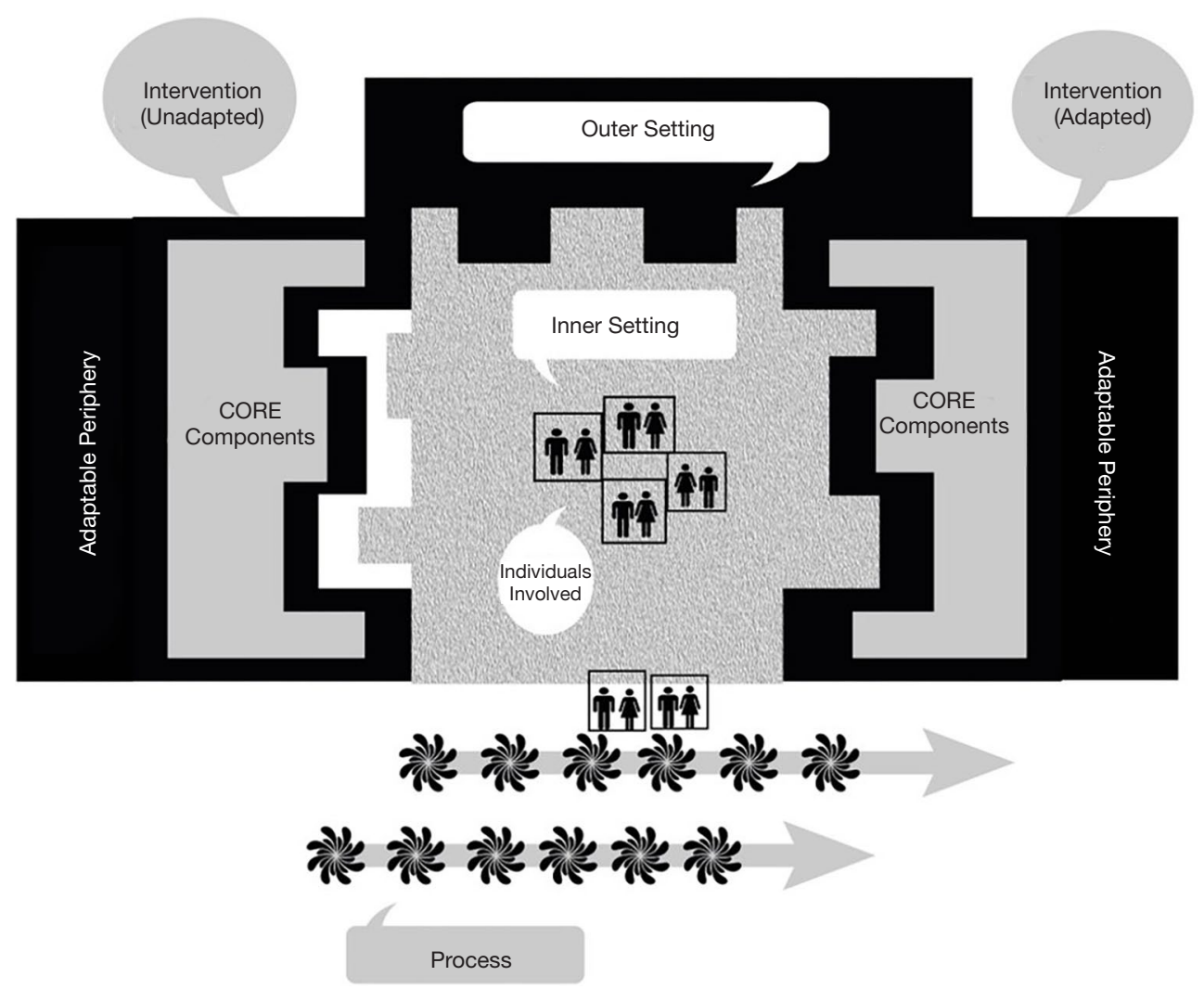

Figure 5 The Consolidated Framework for Implementation Research is an operational framework for evidenced-based intervention to implementation. It identifies constructs among five interacting domains: Intervention, Outer Setting, Inner setting, Individual Characteristics, and Process. The illustration depicts the successful implementation activity from an unadapted intervention (left) to an adapted intervention, [Website: http://cfirguide.org/ (60)].

improving disparate health outcomes. Thus, VB-PHM research in income inequality is a great area of need. This area requires a priority focus in addition to the following areas, including age, gender, race/ethnicity, sexual orientation. Income factors into many aspects of health, such as in each individual's daily choices/lifestyle factors (healthy, fresh produce costs more than going for fast food, and more impoverished families often cannot cover additional costs like gym memberships). Furthermore, more unfortunate patients often cannot afford essential clinic appointments or prescription medicines, leading to having worse health outcomes. Linked to income is also education level, as poorer, less well-educated patients are often less health literate and therefore do not know about regular cancer screenings, the effects of dangerous habits like smoking. Community-based policy focused research provides a link to improving health equity (Figure 4) (64).

\section{Conclusions}

The application of VBPHM has the potential to improve clinical and financial outcomes in the prevention and treatment of cancer and, as a result, improve overall health for a population. Without these efforts, cancer will not only negatively directly impact cancer patients, but all patients as resources get diverted for cancer care. VBPHM is needed now to achieve the Quadruple Aim, which is most critical now in the face of increasing disparities, needs, and decreasing resources. 
Table 5 Applying the RE-AIM and CFIR frameworks in the CBPR planning the evaluation process

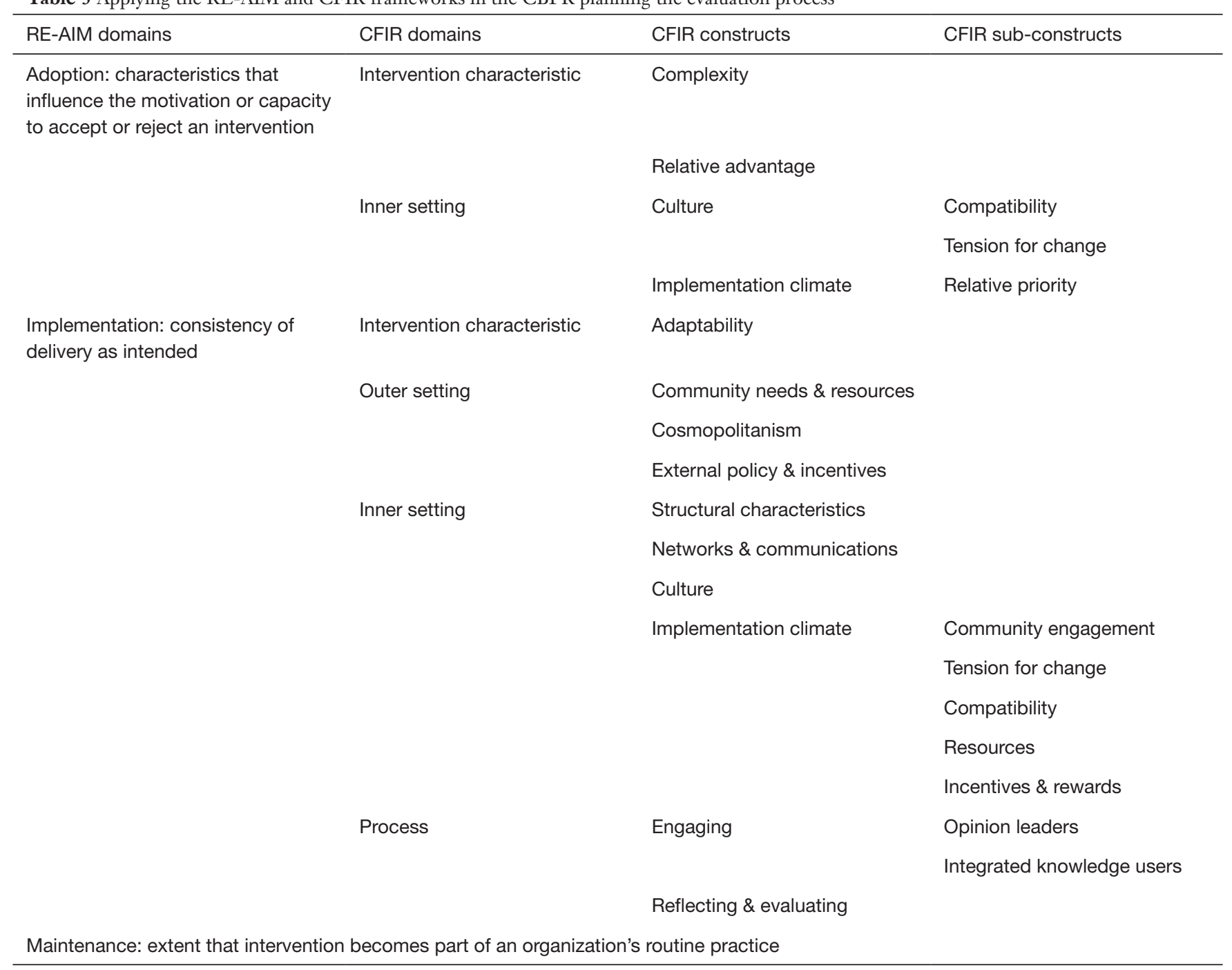

AIM interview questions align with the CFIR domains and constructs $(62,63)$.

\section{Acknowledgments}

The authors wish to recognize the valuable input of Dr. David Kindig as a sounding board for some of the concepts presented in this review. The learners' contributions from the Thomas Jefferson College of Population Health for their thoughtful discussions and critiques of the thoughts generated in the preparation of this study were appreciated. We would like to thank our illustrator, Katelyn Newman, who provided the graphic designs.

Funding: None.

\section{Footnote}

Provenance and Peer Review: This article was commissioned by the editorial office, Fournal of Gastrointestinal Oncology for the series "Global GI Malignancies". The article has undergone external peer review.

Conflicts of Interest: All authors have completed the ICMJE uniform disclosure form (available at http://dx.doi. org/10.21037/jgo-2019-gi-10). The series "Global GI Malignancies" was commissioned by the editorial office 
without any funding or sponsorship. John F. Gibbs served as the unpaid Guest Editor of the series and serves as an unpaid editorial board member of Fournal of Gastrointestinal Oncology from Jan 2019 to Dec 2020. The authors have no other conflicts of interest to declare.

Ethical Statement: The authors are accountable for all aspects of the work in ensuring that questions related to the accuracy or integrity of any part of the work are appropriately investigated and resolved.

Open Access Statement: This is an Open Access article distributed in accordance with the Creative Commons Attribution-NonCommercial-NoDerivs 4.0 International License (CC BY-NC-ND 4.0), which permits the noncommercial replication and distribution of the article with the strict proviso that no changes or edits are made and the original work is properly cited (including links to both the formal publication through the relevant DOI and the license). See: https://creativecommons.org/licenses/by-nc-nd/4.0/.

\section{References}

1. WHO report on cancer: setting priorities, investing wisely, and providing care for all. (2020) Geneva: World Health Organization; 2020. License: CC BY-NC-SA 3.0 IGO.

2. Bray F, Ferlay J, Soerjomataram I, et al. Global Cancer Statistics 2018: GLOBOCAN estimates of incidence and mortality worldwide for 36 cancers in 185 countries. CA Cancer J Clin 2018;68:394-424.

3. Bleakley H. Health, Human Capital, and Development. Annu Rev Econom 2020;2:283-310.

4. The Global Burden of Disease Cancer Collaboration (2015). The Global Burden of Cancer 2013. JAMA Oncol 2015;1:505-27.

5. The global challenge of cancer. (2020). Available online: https://www.nature.com/articles/s43018-019-0023-9

6. Institute of Medicine (US) Committee on Quality of Health Care in America. Crossing the Quality Chasm: A New Health System for the 21st Century. Washington (DC): National Academies Press (US), 2001.

7. National Academies of Sciences, Engineering, and Medicine; Health and Medicine Division; Board on Health Care Services; Board on Global Health; Committee on Improving the Quality of Health Care Globally. Crossing the Global Quality Chasm: Improving Health Care Worldwide. Washington (DC): National Academies Press (US), 2018.
8. Berwick D, Snair M, Nishtar S. Crossing the Global Health Care Quality Chasm: A Key Component of Universal Health Coverage. JAMA 2018;320:1317-8.

9. World Health Organization. (2017). Tackling NCDs: "Best Buys: and other recommended interventions for the prevention and control of noncommunicable diseases. World Health Organization. Available online: https://apps. who.int/iris/handle/10665/259232. License: CC BY-NCSA 3.0 IGO

10. Roberts M, Hsiao W, Berman P, et al. Getting Health Reform Right. Oxford: Oxford University Press, 2002.

11. Morris JN. Uses of Epidemiology. Br Med J 1955;2:395-401.

12. IOM (Institute of Medicine). Population health implications of the Affordable Care Act: Workshop summary. Washington, DC: The National Academies Press, 2014.

13. Kindig D, Stoddart G. What is Population Health? Am J Public Health 2003;93:380-3.

14. Kindig D. What are we talking about when we talk about population health? Health Affairs 2015. doi: 10.1377/ hblog20150406.046151.

15. Berwick DM, Nolan TW, Whittington J. The triple aim: care, health, and cost. Health Aff (Millwood) 2008;27:759-69.

16. Whittington JW, Nolan K, Lewis N, et al. Pursuing the Triple Aim: The First 7 Years. Milbank Q 2015;93:263-300.

17. Bodenheimer T, Sinsky C. From triple to quadruple aim: care of the patient requires care of the provider. Ann Fam Med 2014;12:573-6.

18. Nash DB. The Population Health Mandate: A broader approach to care delivery. BoardRoom Press, February 2012. Available online: https://www.governanceinstitute. com/page/TGIBoardRoomPress

19. Rose G. The Strategy of Preventive Medicine. Oxford: Oxford University Press, 1992.

20. Institute of Medicine (U.S.) Committee on Assuring the Health of the Public in the 21st Century. The Future of the Public's Health in the 21st Century. Washington (D.C.): National Academies Press (U.S.); 2002. 2: Understanding Population Health and Its Determinants. Available online: https://www.ncbi.nlm.nih.gov/books/ NBK221225/

21. Dahlgren G, Whitehead M. Policies and strategies to promote social equity in health. Institute for Futures Studies 1991;14:1-67.

22. Peppard PE, Kindig DA, Dranger E, et al. Ranking community Health Status to Stimulate Discuss of Local 
Public Health Issues: The Wisconsin County Health Ranking. Am J Public Health 2008;98:209-12.

23. Wilkinson RG, Marmot M. (1998). World Health Organization. Regional Office for Europe, WHO Centre for Urban Health (Europe) \& International Centre for Health and Society. Social determinants of health: the solid facts. Available online: https://apps.who.int/iris/ handle/10665/108082

24. Green K, Zook M. When talking about social determinants, precision matters. Health Affairs 2019. doi: 10.1377/hblog20191025.776011.

25. Alderwick H, Gottlieb LM. Meanings and Misunderstandings: A Social Determinants of Health Lexicon for Health Care Systems. Milbank Q 2019;97:407-19.

26. Allen LN, Pullar J, Wickramasinghe KK, et al. Evaluation of research on interventions aligned to WHO 'Best Buys' for NCDs in low-income and lower-middle-income countries: a systematic review from 1990 to 2015 . BMJ Glob Health 2018;3:e000535.

27. Provider-Led Population Health Management: Key Strategies for Healthcare in the Cognitive Era, 2nd Editions. (2016) Richard Hodach, Paul Grundy, Anil Jain, Michael Weiner, John Wiley \& Sons, Inc. Indianapolis IN 46256 2016. Available online: https://www.wiley.com/en-us

28. Nash DB, Fabius RJ, Skoufalos A, et al. Population Health: Creating a Culture of Wellness. 2nd Edition. Burlington, MA: Jones \& Bartlett Learning, 2016.

29. Onukwugha E, Petrelli NJ, Castro KM, et al. Impact of multidisciplinary care on the process of cancer care: A multi-institutional study. J Oncol Pract 2016;12:155-6.

30. Harwood JL, Butler CA, Page AE. Patient-centered and Population Health: Establishing their role in orthopedic practice. J Bone Joint Surg Am 2016;98:e40.

31. Wikipedia contributors. (2020, May 16). County (United States). In: Wikipedia, The Free Encyclopedia. Retrieved 20:22, May 22, 2020. Available online: https:// en.wikipedia.org/w/index.php?title=County_(United_ States)\&oldid=956939701

32. Remington PL, Catlin BB, Gennuso KP. The County Health Rankings: rationale and methods. Popul Health Metr 2015;13:11.

33. County Health Rankings (2019). Available online: https:// www.countyhealthrankings.org/take-action-to-improvehealth

34. Krieger N. Who and what is a "population"? Historical debates, current controversies, and implications for understanding "population health" and rectifying health inequities. Milbank Q 2012;90:634-81.

35. Arndt S, Acion L, Caspers K, et al. How reliable are county and regional health rankings? Prev Sci 2013;14:497-502.

36. Stoto MA, Davis MV, Atkins A. Beyond CHNAS: Performance Measurement for Community Health Improvement. EGEMS (Wash DC) 2019;7:45.

37. Nagasako E, Waterman B, Reidhead M, et al. Measuring Subcounty Differences in Population Health Using Hospital and Census-Derived Data Sets: The Missouri ZIP Health Rankings Project. J Public Health Manag Pract 2018;24:340-9.

38. Athens JK, Remington PL, Gangnon RE. Improving the Rank Precision of Population Health Measures for Small Areas with Longitudinal and Joint Outcome Models. PLoS One 2015; 10:e0130027.

39. Cumberland County NJ (2010). Available online: http:// www.co.cumberland.nj.us/

40. Prioritizing Public Health Problems. Atlanta, GA: Centers for Disease Control and Prevention (CDC), 2013. Available online: https://www.cdc.gov/globalhealth/ healthprotection/fetp/training_modules/4/prioritizeproblems_fg_final_09262013.pdf

41. Braveman P, Egerter S, Williams DR. The social determinants of health: coming of age. Annu Rev Public Health 2011;32:381-98.

42. Thomas-Henkel C, Schulman M. (2017). Screening for social determinants of health in populations with complex needs: Implementation considerations. Available online: http://www.chcs.org/media/SDOH-Complex-CareScreening-Brief-102617.pdf

43. Committee on the Recommended Social and Behavioral Domains and Measures for Electronic Health Records; Board on Population Health and Public Health Practice; Institute of Medicine. Capturing Social and Behavioral Domains and Measures in Electronic Health Records. Washington: National Academies Press, 2015. Available from: https://www.ncbi.nlm.nih.gov/books/NBK268995/

44. Stoto MA. Population Health in the Affordable Care Act Era (2013). Available online: http://www.academyhealth. org/files/AH2013pophealth.pdf

45. Stiefel M, Nolan K. a guide to Measuring the Triple Aim: Population, Experience of Care, and Per Capita Cost. IHI Innovation Series white paper. Cambridge, Massachusetts: Institute for Healthcare Improvement; 2012. (Available on www.IHI.org)

46. Matteotti R, Nurkin S, Gibbs JF. "What is the utility of liver scoring systems in the management of hepatocellular carcinoma (HCC)?" In: Hepato-pancreato-biliary and 
Transplant Surgery: Practical Management of Dilemmas. Available online: https://www.ahpba.org/

47. White AM, Castle IP, Hingson RW, et al. Using Death Certificates to Explore Changes in Alcohol-Related Mortality in the United States, 1999 to 2017. Alcohol Clin Exp Res 2020;44:178-87.

48. Committee on Prevention, Diagnosis, Treatment, and Management of Substance Use Disorders in the U.S. Armed Forces; board on the Health of Select Populations; Institute of Medicine; O'Brien CP, Oster M, Modden E, editors. Substance Use Disorders in the U.S. Armed Forces. Washington (D.C.): National Academies Press (U.S.), 2013. Available online: https://www.nap.edu/

49. Bader TF, Ramon de la Fuente J, Saunders J, Grant M. (1992). AUDIT The Alcohol Use Disorders Identification Test: Guideline for use in Primary Health Care. World Health Organization.

50. Beste LA, Leipertz SL, Green PK, et al. Trends in burden of cirrhosis and hepatocellular carcinoma by underlying liver disease in US veterans, 2001-2013. Gastroenterology 2015;149:1471-e18.

51. Nasir A, Manivel JC, Yousaf H, et al. Markedly Increased Rate of Primary Liver Malignancies at Autopsy in Male US Veterans. Clin Gastroenterol Hepatol 2017;15:316-8.

52. Larson MJ, Mohr BA, Adams RS, et al. Missed opportunity for alcohol problem prevention among army active duty service members postdeployment. Am J Public Health 2014;104:1402-12.

53. Arcaya MC, Arcaya AL, Subramanian SV. Inequalities in health: definitions, concepts, and theories. Glob Health Action 2015;8:27106.

54. Levinsky-Raskin S, Stevens G. "Nothing About Us Without Us." Reflections on Inclusive Practices at Cultural Institutions. Available online: http://ww2.aam-us. org/docs/default-source/resource-library/nothing-aboutus-without-us

55. Jagosh J, Macaulay AC, Pluye P, et al. Uncovering the benefits of participatory research: implications of a realist review for health research and practice. Milbank $\mathrm{Q}$

Cite this article as: Gibbs JF, Newman A, Stefanacci RG. Value-based focused global population health management. J Gastrointest Oncol 2021;12(Suppl 2):S275-S289. doi: 10.21037/jgo-2019-gi-10
2012;90:311-46.

56. Israel BA, Schulz AJ, Parker EA, et al. Review of community-based research: assessing partnership approaches to improve public health. Annu Rev Public Health 1998;19:173-202.

57. Salsberg J, Macaulay AC, Parry D. Guide to Integrated Knowledge Translation Research. In: Graham ID, Tetroe J, Pearson A. editors. Turning Knowledge into Action: Practical Guidance on How to Do Integrated Knowledge Translation Research. Adelaide: Wolters Kluwer-Joanna Briggs Institute, 2014:176-82.

58. Makosky Daley C, James AS, Ulrey E, et al. Using focus groups in community-based participatory research: challenges and resolutions. Qual Health Res 2010;20:697-706.

59. Smith SA, Blumenthal DS. Community health workers support community-based participatory research ethics: lessons learned along the research-to-practice-tocommunity continuum. J Health Care Poor Underserved 2012;23:77-87.

60. Damschroder LJ, Aron DC, Keith RE, et al. Fostering implementation of health services research findings into practice: a consolidated framework for advancing implementation science. Implement Sci 2009;4:50.

61. Glasgow RE, Vogt TM, Boles SM. Evaluating the public health impact of health promotion interventions: the REAIM framework. Am J Public Health 1999;89:1322-7.

62. King DK, Shoup JA, Raebel MA, et al. Planning for Implementation Success Using RE-AIM and CFIR Frameworks: A Qualitative Study. Front Public Health 2020;8:59.

63. Green HD. A Community-Based Evaluation of Screening, Brief Intervention, and Referral to Treatment (SBIRT) for the Black Community. Qual Health Res 2018;28:418-32.

64. Cacari-Stone L, Wallerstein N, Garcia AP, et al. The promise of community-based participatory research for health equity: a conceptual model for bridging evidence with policy. Am J Public Health 2014;104:1615-23. 